\title{
Thermal state modeling in thermosensitive elements of microelectronic devices with reach-through foreign inclusions
}

\author{
V.I. Gavrysh \\ National University "Lviv Polytechnic”, 28-a, S. Bandery str., 79013 Lviv, Ukraine \\ E-mail: ikni.pz@gmail.com
}

\begin{abstract}
The nonlinear boundary axially symmetric problem of heat conduction for the thermosensitive piecewise homogeneous layer with reach-through cylindrical inclusion that generates heat has been considered. Using the introduced function, the partial linearization of the original problem has been carried out. With the proposed piecewiselinear approximation of temperature at the boundary surface of the foreign inclusion and on the contact surface of the homogeneous elements of the layer, the problem has been completely linearized. The analytical solution of this problem of finding the introduced function using Hankel integral transform has been formed. The formulae for calculating the desired temperature have been derived.
\end{abstract}

Keywords: temperature, thermal conductivity, steady-state, ideal thermal contact, foreign reach-through inclusion, thermosensitive.

Manuscript received 08.12.11; revised version received 15.05.12; accepted for publication 14.06.12; published online 25.09.12.

\section{Introduction}

For microelectronic devices, the light-emitting elements based on organic materials are commonly developed. The basis for such a device is an organic microparticle with electroluminescent properties, i.e. when it is stimulated by current, it emits light. Due to it, the mentioned elements based on organic materials reflecting the random color will be very thin and will be implemented on flexible substrates. Using two vacuum units of thermovacuum spraying, from one of them a thin film structure of organic materials is formed, and from another - electrodes. In such a combination, ready LED is obtained. To improve the efficiency of organic LEDs, as better and stable operation parameters (brightness, time of operation, reliability, etc.), the effect of large temperature gradients and absolute values of temperature should be taken into account.

Some researches of the temperature conditions for nodes and separate elements of microelectronic devices have been made previously [1-8].
Hereinafter the boundary axially symmetric problem of heat conduction for a single element or node of microelectronic devices that is modulated with thermosensitive piecewise homogeneous layer with heatgenerating reach-through foreign cylindrical inclusion has been considered.

\section{Formulation of the problem}

Let us consider an isotropic, in the sense of thermophysical properties, thermosensitive piecewisehomogeneous layer, which consists of $n$ homogeneous elements that differ in geometric and thermophysical parameters, and which is assigned to a cylindrical coordinate system (Or $\varphi z)$ with the beginning on one of its edges, and it contains reach-through foreign inclusion with the radius $R$. On the contact surfaces

$$
\begin{aligned}
& S_{R}=\{(R, \varphi, z): 0 \leq \varphi \leq 2 \pi\}, \\
& S_{i}=\left\{\left(r, \varphi, z_{i}\right): r>R, 0 \leq \varphi \leq 2 \pi, i=\overline{1, n-1}\right\},
\end{aligned}
$$


where $z_{i}$ is the thickness of the $i$-th element layer, the ideal thermal contact takes place.

In the region

$\Omega_{0}=\left\{(r, \varphi, z): r \leq R, 0 \leq \varphi \leq 2 \pi, 0 \leq z \leq z_{n}\right\}$,

of inclusion, the uniformly distributed internal heat sources with the capacity $q_{0}$ have influence. On the boundary surfaces of the layer $K_{0}=\{(r, \varphi, 0): r<\infty, \quad 0 \leq \varphi \leq 2 \pi\}$, $K_{n}=\left\{\left(r, \varphi, z_{n}\right): r<\infty, \quad 0 \leq \varphi \leq 2 \pi\right\}, \quad$ the boundary conditions of the second kind are set (Figure).

\section{Construction of partially linear mathematical model}

The distribution of steady state axially symmetric temperature field $t(r, z)$ in the system under consideration is obtained by solving the nonlinear heat equation $[9,10]$

$\frac{1}{r} \frac{\partial}{\partial r}\left[r \lambda(r, z, t) \frac{\partial t}{\partial r}\right]+\frac{\partial}{\partial z}\left[\lambda(r, z, t) \frac{\partial t}{\partial z}\right]=$

$=-q_{0} S_{-}(R-r)$

with the following boundary conditions

$\left.t\right|_{r \rightarrow \infty}=0,\left.\frac{\partial t}{\partial r}\right|_{r \rightarrow \infty}=\left.\frac{\partial t}{\partial z}\right|_{z=0}=\left.\frac{\partial t}{\partial z}\right|_{z=z_{n}}=0$,

where

$\lambda(r, z, t)=\sum_{i=1}^{n}\left\{\lambda_{i}(t)+\left[\lambda_{0}(t)-\lambda_{i}(t)\right] \cdot S_{-}(R-r)\right\} \cdot N\left(z, z_{i-1}\right)$ is the thermal conductivity coefficient of piecewisehomogeneous layer; $\lambda_{i}(t), \lambda_{0}(t)-$ thermal conductivity coefficients of materials of $i$-th layer and inclusion, respectively;

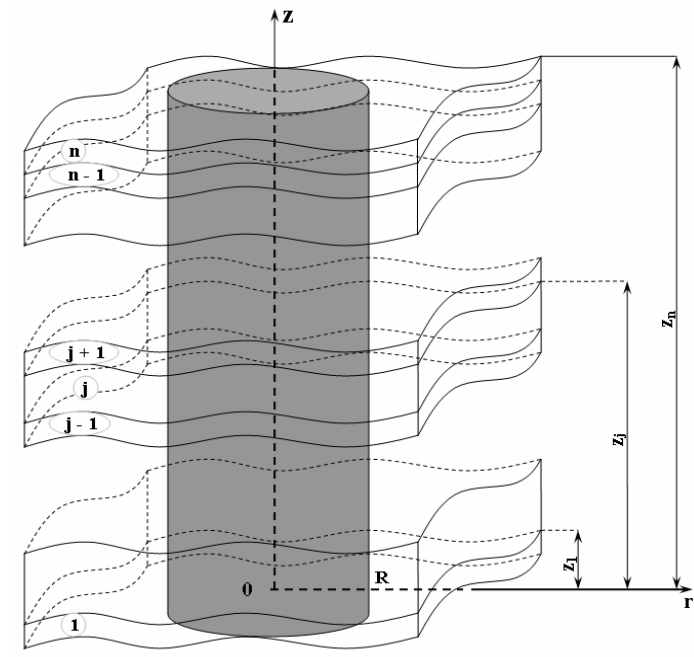

Thermosensitive isotropic piecewise homogeneous layer with heat generating reach-through foreign cylindrical inclusion. $z_{0}=0 ; N\left(z, z_{i-1}\right)=S_{+}\left(z-z_{i-1}\right)-S_{+}\left(z-z_{i}\right) ;$

$S_{ \pm}(\zeta)= \begin{cases}1, & \zeta>0 \\ 0,5 \mp 0,5, \zeta & =0-\text { asymmetric unit function } \\ 0, & \zeta<0\end{cases}$

[11].

Let us introduce the function

$\vartheta=\sum_{i=1}^{n}\left\{\int_{0}^{t(r, z)} \lambda_{i}(\zeta) d \zeta \cdot N\left(z, z_{i-1}\right)+S_{-}(R-r)\left[N\left(z, z_{i-1}\right)\right.\right.$

$\int_{t(R, z)}^{t(r, z)}\left(\lambda_{0}(\zeta)-\lambda_{i}(\zeta)\right) d \zeta-\int_{t\left(R, z_{i-1}\right)}^{t\left(r, z_{i-1}\right)}\left(\lambda_{0}(\zeta)-\lambda_{i}(\zeta)\right) d \zeta \cdot S_{+}\left(z-z_{i-1}\right)+$

$\left.+\int_{t\left(R, z_{i}\right)}^{t\left(r, z_{i}\right)}\left(\lambda_{0}(\zeta)-\lambda_{i}(\zeta)\right) d \zeta \cdot S_{+}\left(z-z_{i}\right)\right]-\int_{0}^{t\left(r, z_{i-1}\right)} \lambda_{i}(\zeta) d \zeta \cdot S_{+}\left(z-z_{i-1}\right)$

$\left.\begin{array}{r}t\left(r, z_{i}\right) \\ +\quad \int_{0} \lambda_{i}(\zeta) d \zeta \cdot S_{+}\left(z-z_{i}\right)\end{array}\right\}$

by differentiation of which with respect to $r$ and $z$ we obtain

$\lambda(r, z, t) \frac{\partial t}{\partial r}=\frac{\partial \vartheta}{\partial r}+F_{1}(r, z)$
$\lambda(r, z, t) \frac{\partial t}{\partial z}=\frac{\partial \vartheta}{\partial z}+F_{2}(r, z)$

where

$F_{1}(r, z)=\sum_{i=1}^{n}\left[\left.\left(\lambda_{i}(t) \frac{\partial t}{\partial r}\right)\right|_{z=z_{i-1}} \times\right.$

$\left.\times S_{+}\left(z-z_{i-1}\right)-\left.\left(\lambda_{i}(t) \frac{\partial t}{\partial r}\right)\right|_{z=z_{i}} \cdot S_{+}\left(z-z_{i}\right)\right]$,

$F_{2}(r, z)=\left.S_{-}(R-r) \cdot \sum_{i=1}^{n}\left[\left(\lambda_{0}(t)-\lambda_{i}(t)\right) \frac{\partial t}{\partial z}\right]\right|_{r=R} \cdot N\left(z, z_{i-1}\right)$.

Taking into account the expressions (4), the original equation (1) takes the following form:

$\frac{1}{r} \frac{\partial}{\partial r}\left[r \frac{\partial \vartheta}{\partial r}\right]+\frac{\partial^{2} \vartheta}{\partial z^{2}}+\frac{1}{r} \frac{\partial}{\partial r}\left[r \cdot F_{1}(r, z)\right]+$

$+\frac{\partial}{\partial z}\left[F_{2}(r, z)\right]=-q_{0} \cdot S_{-}(R-r)$.

Using the equation (3) the boundary conditions can be written as:

$\vartheta\left|=0, \frac{\partial \vartheta}{\partial r}\right|_{r \rightarrow \infty}=\left.\frac{\partial \vartheta}{\partial z}\right|_{z=0}=\left.\frac{\partial \vartheta}{\partial z}\right|_{z=z_{n}}=0$.

Thus, the introduced function $\vartheta$ represented by the expression (3) has allowed to convert the non-linear heat conduction problem (1), (2) to partially linear equations with discontinuous coefficients (5) and completely linear boundary conditions (6). 


\section{Absolutely linearized mathematical model}

Let the approximate functions $t(R, z), t\left(r, z_{i}\right)$ look as

$t(R, z)=t_{1}^{(i R)}+\sum_{k=1}^{m-1}\left(t_{k+1}^{(i R)}-t_{k}^{(i R)}\right) S_{-}\left(z-z_{k}^{(i)^{*}}\right)$,

$t\left(r, z_{i}\right)=t_{1}^{(i)}+\sum_{j=1}^{l-1}\left(t_{j+1}^{(i)}-t_{j}^{(i)}\right) S_{-}\left(r-r_{j}\right)$,

where

$\left.z_{k}^{(i)^{*}} \in\right] 0 ; z_{i}\left[; z_{1}^{(i)^{*}} \leq z_{2}^{(i)^{*}} \leq \ldots \leq z_{m-1}^{(i)^{*}} ;\right.$

$\left.r_{j} \in\right] R ; \quad r_{*}\left[; \quad r_{1} \leq r_{2} \leq \ldots \leq r_{l-1} ; l, m-\quad\right.$ number of fragmentation of intervals $] R ; r_{*}[$ and $] 0 ; z_{i}[$ respectively;

$t_{k}^{(i R)}(k=\overline{1, m-1}), t_{j}^{(i)}(j=\overline{1, l-1})-$ unknown

approximated temperature values; $r_{*}-$ value of radial coordinate, where the temperature practically equals to zero (is found from the corresponding linear model).

Substituting the expression (7) into the equation (5), we obtain the linear differential equation with partial derivatives relative to the introduced function $\vartheta$

$$
\begin{aligned}
& \Delta \vartheta=-\sum_{i=1}^{n}\left[\frac{1}{r} \sum_{j=1}^{l-1} F_{i}^{(j)}(z) \delta_{-}^{\prime}\left(r-r_{j}\right)+S_{-}(R-r) \times\right. \\
& \left.\times \sum_{k=1}^{m-1} F_{i}^{(k)}(z)\right]-q_{0} S_{-}(R-r) .
\end{aligned}
$$

Here

$F_{i}^{(k)}(z)=\left(t_{k+1}^{(i R)}-t_{k}^{(i R)}\right)\left[\lambda_{0}\left(t_{k+1}^{(i R)}\right)-\lambda_{i}\left(t_{k+1}^{(i R)}\right)\right] \cdot \delta_{-}^{\prime}\left(z-z_{k}^{(i)^{*}}\right) ;$

$F_{i}^{(j)}(z)=r_{j}\left[\left(t_{j+1}^{(i-1)}-t_{j}^{(i-1)}\right) \cdot \lambda_{i}\left(t_{j+1}^{(i-1)}\right) \cdot S_{+}\left(z-z_{i-1}\right)-\right.$

$\left.-\left(t_{j+1}^{(i)}-t_{j}^{(i)}\right) \cdot \lambda_{i}\left(t_{j+1}^{(i)}\right) \cdot S_{+}\left(z-z_{i}\right)\right]$;

$\Delta=\frac{1}{r} \frac{\partial}{\partial r}\left(r \frac{\partial}{\partial r}\right)+\frac{\partial^{2}}{\partial z^{2}}$ - Laplace operator in the

cylindrical coordinate system;

$\delta_{ \pm}(\zeta)=\frac{d S_{ \pm}(\zeta)}{d \zeta}-$ asymmetric Dirac delta function [11]

\section{Construction of the analytical solution for the boundary value problem (8), (6)}

Applying the Hankel integral transform by the coordinate $r$ to the equation (8) and boundary conditions (6), we obtain the ordinary differential equations with constant coefficients

$$
\begin{aligned}
& \frac{d^{2} \bar{\vartheta}}{d z^{2}}-\xi^{2} \bar{\vartheta}=-\sum_{i=1}^{n}\left[\xi \sum_{j=1}^{l-1} J_{1}\left(r_{j} \xi\right) \cdot F_{i}^{(j)}(z)+\right. \\
& \left.+\frac{R}{\xi} J_{1}(R \xi) \cdot \sum_{k=1}^{m-1} F_{i}^{(k)}(z)\right]-\frac{q_{0} R}{\xi} J_{1}(R \xi)
\end{aligned}
$$

and boundary conditions

$$
\left.\frac{d \bar{\vartheta}}{d z}\right|_{z=0}=\left.\frac{d \bar{\vartheta}}{d z}\right|_{z=z_{n}}=0
$$

where $\bar{\vartheta}=\int_{0}^{\infty} r J_{0}(r \xi) \vartheta d r$ is the transformant of the function $\vartheta(r, z) ; \quad \xi-\quad$ central Hankel transform parameter; $J_{v}(\zeta)$ - Bessel function of the first kind of $v$-th order.

Let us rewrite the above general view of Eq. (9) in the following form

$$
\begin{aligned}
& \bar{\vartheta}=c_{1} e^{\xi z}+c_{2} e^{-\xi z}+\frac{1}{\xi}\left\{\sum _ { i = 1 } ^ { n } \left[\sum_{j=1}^{l-1} r_{j} J_{1}\left(r_{j} \xi\right) \times\right.\right. \\
& \times\left(\left(1-\operatorname{ch} \xi\left(z-z_{i-1}\right)\right) S_{+}\left(z-z_{i-1}\right) \cdot\left(t_{j+1}^{(i-1)}-t_{j}^{(i-1)}\right) \lambda_{i}\left(t_{j+1}^{(i-1)}\right)-\right. \\
& \left.-\left(1-\operatorname{ch} \xi\left(z-z_{i}\right)\right) S_{+}\left(z-z_{i}\right) \cdot\left(t_{j+1}^{(i)}-t_{j}^{(i)}\right) \cdot \lambda_{i}\left(t_{j+1}^{(i)}\right)\right)- \\
& -R J_{1}(R \xi) \cdot \sum_{k=1}^{m-1}\left(t_{k+1}^{(i R)}-t_{k}^{(i R)}\right) \cdot\left(\lambda_{0}\left(t_{k+1}^{(i R)}\right)-\right. \\
& \left.\left.\left.-\lambda_{i}\left(t_{k+1}^{(i R)}\right)\right) \cdot \operatorname{ch} \xi\left(z-z_{k}^{(i)^{*}}\right) S_{-}\left(z-z_{k}^{(i)^{*}}\right)\right]+\frac{q_{0} R}{\xi^{2}} J_{1}(R \xi)\right\} .
\end{aligned}
$$

Here, $c_{1}, c_{2}$ - integration constants.

Using the boundary conditions (10), we obtain a partial solution to the problem (9), (10):

$$
\begin{aligned}
& \bar{\vartheta}=\frac{1}{\xi}\left\{\sum _ { i = 1 } ^ { n } \left[\sum _ { j = 1 } ^ { l - 1 } r _ { j } J _ { 1 } ( r _ { j } \xi ) \cdot \left(\left(\left(1-\operatorname{ch} \xi\left(z-z_{i-1}\right)\right) S_{+}\left(z-z_{i-1}\right)+\right.\right.\right.\right. \\
& \left.+\frac{\operatorname{ch} \xi z}{\operatorname{sh} \xi z_{n}} \operatorname{sh} \xi\left(z_{n}-z_{i-1}\right)\right) \cdot\left(t_{j+1}^{(i-1)}-t_{j}^{(i-1)}\right) \lambda_{i}\left(t_{j+1}^{(i-1)}\right)- \\
& -\left(\left(1-\operatorname{ch} \xi\left(z-z_{i}\right)\right) S_{+}\left(z-z_{i}\right)+\frac{\operatorname{ch} \xi z}{\operatorname{sh} \xi z_{n}} \operatorname{sh} \xi\left(z_{n}-z_{i}\right)\right) \times \\
& \left.\left.\times\left(t_{j+1}^{(i)}-t_{j}^{(i)}\right) \lambda_{i}\left(t_{j+1}^{(i)}\right)\right)\right)-R J_{1}(R \xi) \cdot \sum_{k=1}^{m-1}\left(t_{k+1}^{(i R)}-t_{k}^{(i R)}\right) \times \\
& \times\left(\lambda_{0}\left(t_{k+1}^{(i R)}\right)-\lambda_{i}\left(t_{k+1}^{(i R)}\right)\right)\left(\operatorname{ch} \xi\left(z-z_{k}^{(i)}\right)^{*}\right) \cdot S_{-}\left(z-z_{k}^{(i)^{*}}\right)- \\
& \left.\left.\left.-\frac{\operatorname{ch} \xi z}{\operatorname{sh} \xi z_{n}} \operatorname{sh} \xi\left(z_{n}-z_{k}^{(i)}\right)\right)\right]+\frac{q_{0} R}{\xi^{2}} J_{1}(R \xi)\right\} .
\end{aligned}
$$

Applying the inverse Hankel integral transform to the equation (11), we find the expression for the function $\vartheta$

$$
\vartheta=\int_{0}^{\infty} \xi J_{0}(r \xi) \cdot \bar{\vartheta} d \xi
$$

By substitution of the specific dependence of thermal conductivity coefficients of materials of each 
element layer and inclusion in relations (3), (12) and by comparison of the obtained expressions for the function $\vartheta$ on surfaces $S_{R}, S_{i}(i=\overline{1, n-1})$, we come to systems of nonlinear algebraic equations for determination of the unknown temperature values $t_{k}^{(i R)}(k=\overline{1, m}) \quad$ and $t_{j}^{(i)}(j=\overline{1, l})$.

The desired temperature field for the considered system is determined from the nonlinear algebraic equations obtained using the relations (3), (12) after substituting to them the specific expression of dependences of the thermal conductivity coefficients for material elements of the layer and inclusion.

\section{Partial illustration and analysis of the results obtained}

In many practical cases, there is such dependence of the thermal conductivity coefficient on temperature $[11,12]$ :

$\lambda=\lambda^{0}(1-k t)$,

where $\lambda^{0}, k$ are pivotal and temperature coefficients of thermal conductivity.

Then, using Exps (3) and (12) yields a formula for determining the temperature $t$ for the case of 2-D layer $(n=2)$ in the areas

$\Omega_{1}=\left\{(r, \varphi, z): r>R, 0 \leq \varphi \leq 2 \pi, 0 \leq z<z_{1}\right\}$,

$t=\frac{1-\sqrt{1-2 \frac{k_{1}}{\lambda_{1}^{0}}\left(\vartheta+\vartheta_{1}\right)}}{k_{1}} ;$

$\Omega_{2}=\left\{(r, \varphi, z): r>R, 0 \leq \varphi \leq 2 \pi, z_{1} \leq z \leq z_{2}\right\}$,

$t=\frac{1-\sqrt{1-2 \frac{k_{2}}{\lambda_{2}^{0}}\left(\vartheta+\vartheta_{2}\right)}}{k_{2}}$;

$\Omega_{3}=\left\{(r, \varphi, z): r \leq R, 0 \leq \varphi \leq 2 \pi, 0 \leq z<z_{1}\right\}$,

$t=\frac{1-\sqrt{1-2 \frac{k_{0}}{\lambda_{0}^{0}}\left(\vartheta+\vartheta_{3}\right)}}{k_{0}} ;$

$\Omega_{4}=\left\{(r, \varphi, z): r \leq R, 0 \leq \varphi \leq 2 \pi, z_{1} \leq z \leq z_{2}\right\}$,

$t=\frac{1-\sqrt{1-2 \frac{k_{0}}{\lambda_{0}^{0}}\left(\vartheta+\vartheta_{4}\right)}}{k_{0}}$.

Here, $\vartheta_{1}=\left.\lambda_{1}^{0}\left[\left(1-\frac{k_{1}}{2} t\right) t\right]\right|_{z=0}$;

$\vartheta_{2}=\vartheta_{m}+\vartheta_{1} ; \quad \vartheta_{m}=\left.\left[\left(\lambda_{2}^{0}-\lambda_{1}^{0}+\frac{\lambda_{1}^{0} k_{1}-\lambda_{2}^{0} k_{2}}{2} t\right) t\right]\right|_{z=z}$; $\vartheta_{3}=\vartheta_{v}^{(1)}-\left.\vartheta_{v}^{(1)}\right|_{z=0}+\vartheta_{0} ; \vartheta_{0}=\left.\lambda_{0}^{0}\left[\left(1-\frac{k_{0}}{2} t\right) t\right]\right|_{z=0} ;$

$\vartheta_{v}^{(i)}=\left[\lambda_{0}^{0}-\lambda_{i}^{0}+\left(\frac{\lambda_{i}^{0} k_{i}-\lambda_{0}^{0} k_{0}}{2} t\right) t \|_{r=R} i=1,2\right.$;

$\vartheta_{4}=\vartheta_{v}^{(2)}-\left.\vartheta_{v}^{(1)}\right|_{z=0}+\left.\vartheta_{m}\right|_{r=R}+\vartheta_{0} ; \quad$ the value of temperature $t(r, 0)$ is such that is equal to the environment temperature, $t(R, z), t\left(R, z_{1}\right)$ are calculated using the formula (14).

\section{Conclusions}

The introduced function $\vartheta$ described by the expression (3) allowed to partially linearize the original nonlinear heat equation (1) and completely linearize the boundary conditions (2). The proposed piecewise-linear approximation of the temperature expressions (7) on the boundary surface $S_{R}$ of the foreign inclusion and of contact surfaces $S_{i}(i=\overline{1, n-1)}$ for homogeneous elements of the layer gave the opportunity to completely linearize the equation (5). And therefore, it was possible to apply the Hankel integral transform to the obtained boundary linear problem to the introduced function $\vartheta$ and to construct an analytical solution for finding it. The 2-D layer with the dependence of the thermal conductivity coefficient of the layers and inclusion described by the expressions (13) has been considered. On this basis, the formulae (14)-(17) for the calculation of the temperature $t(r, z)$ at any point of the considered system have been shown.

\section{References}

1. A.F. Barvinskyy, V.I. Gavrysh, Nonlinear thermal conductivity problem for nonhomogeneous layer with internal heat sources // Journal of Mechanical Engineering, 12(1), p. 47-53 (2009) in Ukrainian.

2. V.I. Gavrysh, D.V. Fedasyuk, The method for calculation of temperature fields for thermosensitive piecewise homogeneous strip with foreign inclusion // Industrial Heat Engineering, 32(5), p. 18-25 (2010) in Ukrainian.

3. V.I. Gavrysh, D.V. Fedasyuk, A.I. Kosach, Boundary thermal conductivity problem for the layer with cylindrical inclusion // Physicochemical Mechanics of Materials, 46(5), p. 115-120 (2010), in Ukrainian.

4. V.I. Gavrysh, D.V. Fedasyuk, Thermal simulation of heterogeneous structural components in microelectronic devices // Semiconductor Physics, Quantum Electronics \& Optoelectronics, 13, No.4, p. 439-443 (2010).

5. V.I. Gavrysh, A.I. Kosach, Simulation of temperature conditions in the elements of 
microelectronic devices // Tekhnologiya $i$ konstruirovanie v elektron. apparature, №1-2 (90), p. 27-30 (2011) in Russian.

6. V.I. Gavrysh, A.I. Kosach, Boundary thermal conductivity problem for the layer with cylindrical inclusion // Physicochemical Mechanics of Materials 47(6), p. $52-58$ (2011) in Ukrainian.

7. V.I. Gavrysh, Thermal simulation of heterogeneous structural components in microelectronic devices // Semiconductor Physics, Quantum Electronics \& Optoelectronics, 14(4), p. 478-481 (2011).

8. V.I. Gavrysh, A.I. Kosach, Simulation of temperature conditions in electronic devices of piecewise-homogeneous structure // Electronic Modeling, 33(4), p. 99-113 (2011) in Russian.
9. Ya.S. Podstryhach, V.A. Lomakin, Yu.M. Kolyano, Thermoelasticity of Heterogeneous Body Structure. Nauka, Moscow, 1984 (in Russian).

10. Yu.M. Kolyano, Methods of Heat Conductivity and Thermoelasticity of Heterogeneous Bodies. Kyiv, Naukova Dumka, 1992 (in Russian).

11. G. Korn, T. Korn, Mathematical Handbook for Scientists and Engineers. Nauka, Moscow, 1977 (in Russian).

12. V.A. Lomakin, Theory of Elasticity of Heterogeneous Bodies. Moscow University Publishing House, 1976 (in Russian).

13. R. Berman, Thermal Conductivity of Solids. Mir, Moscow, 1979 (in Russian). 